\title{
Reduced Beige Adipogenic Potential in Subcutaneous Adipocytes Derived from Obese Chinese Individuals
}

This article was published in the following Dove Press journal: Diabetes, Metabolic Syndrome and Obesity: Targets and Therapy

\author{
Han $\mathrm{Li}^{1, *}$ \\ Lin Shen ${ }^{2, *}$ \\ Lei Zhang ${ }^{3}$ \\ Bing $\mathrm{Yan}^{3}$ \\ Tao Sun ${ }^{3}$ \\ Feng Guo ${ }^{3}$ \\ Xiao Yin' \\ 'Department of Endocrinology, Jinan \\ Central Hospital Affiliated to Shandong \\ University, Jinan, Shandong 2500I3, \\ People's Republic of China; ${ }^{2}$ Shandong \\ First Medical University, Taian, Shandong \\ 27I000, People's Republic of China; \\ ${ }^{3}$ Department of Surgery, Jinan Central \\ Hospital Affiliated to Shandong \\ University, Jinan, Shandong 2500I3, \\ People's Republic of China \\ *These authors contributed equally to \\ this work
}

Correspondence: Xiao Yin Email yinxiao@sdu.edu.cn
Purpose: Thermogenesis function has made brown/beige adipocyte an attractive target for obesity. Human brown adipose tissue activity is impaired in obesity in vivo. The present study aims to compare the differences in beige adipocyte differentiation potential of subcutaneous adipose tissue derived from normal weight and obese Chinese individuals in vitro. Methods: Adipose-derived stem cells (ADSCs) isolated from subcutaneous fat tissues of normal weight $(\mathrm{NW})$ and obese $(\mathrm{OB})$ groups were induced to differentiate into mature adipocyte with white adipocyte (WA)- and beige adipocyte (BA)-induction treatment. The expression of beige adipocyte marker protein UCP-1 and specific thermogenic genes was detected in differentiated adipocytes via Western blot and rt PCR, and the adipocyte mitochondrial function and lipolysis ability were also measured by oxygen consumption rate (OCR) and glycerol release rate, respectively.

Results: Either with WA-induction or BA-induction, the expression of UCP-1 and beige adipocyte-specific thermogenic genes in differentiated adipocytes was higher in the NW compared to the OB group, followed by higher OCR and lipolysis ability in NW group than OB group. With BAinduction, expression of UCP-1 and thermogenic genes increased significantly, followed by the increasement in adipocytes OCR and lipolysis rate in NW group compared with WA-induction treatment, but no significant difference was observed in OB group.

Conclusion: Compromised beige adipocyte differentiation plasticity was found in subcutaneous white adipose tissue derived from obese Chinese individuals, which may be due part to the downregulation of $\beta 3$-adrenergic receptor expression in adipocytes. Discovery of therapeutic agents to active brown adipose tissue through specific pathways could provide a promising approach for treating obesity in the future.

Keywords: obesity, beige adipocyte, subcutaneous fat, Chinese

\section{Introduction}

The worldwide occurrence of obesity and its comorbidities continues to rise along with a demand for novel therapeutic interventions. ${ }^{1}$ As the main site of non-shivering thermogenesis and oxidation of glucose as well as lipids, ${ }^{2}$ the diminished human brown adipose tissue (hBAT) activity may account for some types of obesity and it has been regarded as the target to combat obesity simultaneously. BAT was composed of classical brown adipocytes, which was characterized by enrichment mitochondria and expression of marker protein UCP-1. UCP-1 protein locates in the inner membrane of mitochondria, diminishing the proton gradient and releasing energy as heat. ${ }^{3}$ Classical brown adipocytes rarely exist in adults, but beige adipocytes in white adipose tissue (WAT) also express 
UCP-1 and possess the same thermogenic function as brown adipocyte when facing some stimuli, which provides an available strategy to induce thermogenic adipocytes to increase the activation of hBAT. Previous human in vivo studies have evaluated hBAT activity using 18-fluorodeoxyglucose-PET$\mathrm{CT}$ and found that lean body mass is positively related to hBAT activity, ${ }^{4,5}$ but did not elucidate the difference of beige adipogenic potential at the molecular level in adipocytes derived from persons with different adiposity, and the data concerning the obese Chinese population in this field was especially scarce. Thus, in this study, we recruited normal weight and obese Chinese individuals, and examined the beige adipocyte induction potential at molecular and cellular function levels through primary human adipocytes culture and white adipocyte (WA)-/beige adipocyte (BA)-induction treatment, which could provide comprehensive information about adipose tissue browning capability in persons with different adiposity and the possible underling mechanism involved.

\section{Materials and Methods}

\section{Participants}

Human subcutaneous adipose tissues were collected from $35-60$-year-old donors ( $n=24$, with BMI $>28$ or BMI $<24$ ) who underwent selective plastic surgery in Jinan Central Hospital. Exclusion criteria include cancer, thyroid diseases, diabetes, serious infectious diseases and patients who have been taking thyroxine, insulin, thiazolidinedione, lipidlowering medicine or GLP-1 analogues in the last 3 months. Height, weight, waist circumference and blood pressure were measured before surgery. Body mass index (BMI) was calculated as weight (kilograms) divided by height (meters) squared. Participants were divided into two groups according to their BMI: normal-weight (NW) group (BMI $<24 \mathrm{~kg} / \mathrm{m}^{2}$ ), obese $(\mathrm{OB})$ group $\left(\mathrm{BMI}>28 \mathrm{~kg} / \mathrm{m}^{2}\right)$. All participants signed informed consent. This experiment was conformed with the provisions of the Declaration of Helsinki and was approved by the local Ethical and Research Committee of Jinan Central Hospital (Shandong, China). The ethical number is 201,907,702.

\section{Resting Metabolic Rate, Biochemical Parameters and Abdominal Fat Area Measurement}

Resting metabolic rate (RMR) was measured using a ventilated hood coupled with an open-circuit indirect calorimeter. Every participant's RMR was measured in the early morning, relaxing. Respiratory exchange data from the final $10 \mathrm{~min}$ of the 30 -min data collection period were used to calculate RMR.

Blood samples were collected in the morning after 10 hours of fasting to determine biochemical parameters, including HDL-C, triglycerides (TG), LDL-C and total cholesterol (TC), using an automatic analyzer (Hitachi 7080, Tokyo, Japan).

Abdominal subcutaneous fat area (SFA) and visceral fat area (VFA) were measured via bioelectrical impedance analysis (BIA) (Tanita BC 420MA/SC-331S, Tokyo, Japan).

\section{Immunohistochemistry}

Fresh adipose tissue samples were fixed, embedded in paraffin and cut into slices, then deparaffinized and the antigen retrieved at $95^{\circ} \mathrm{C}$. Tissue sections were incubated with antibodies against UCP-1 (1:100) overnight at $4{ }^{\circ} \mathrm{C}$, then with horseradish peroxidase (HRP)-conjugated antirabbit secondary antibody for 1 hour at room temperature, stained with DAB to detect the images and take photos by inverted phase contrast microscope (Zeiss Axiovert A1, Germany).

\section{Primary Adipocyte Culture and Adipogenic Induction}

Freshly dissected adipose tissues were digested in buffer contained collagenase $(1 \mathrm{mg} / \mathrm{mL})$ for 30 minutes, human adipose-derived stem cells (ADSCs) was isolated by centrifugation (1200 rpm, $10 \mathrm{~min}$ ) and cultured in basic medium (DMEM/Ham's F-12 containing 10\% fetal bovine serum (Sigma-Aldrich), 1\% penicillin-streptomycin, and $0.01 \%$ fibroblast growth factor) to obtain preadipocytes. ${ }^{6}$

For WA-induction $(n=24)$, the preadipocytes were cultured in WA-induction medium (basic medium supplemented with $1 \mu \mathrm{M}$ dexamethasone, $500 \mu \mathrm{M}$ 3-isobutyl-1-methylxanthine (IBMX), $0.85 \mu \mathrm{M}$ insulin, $200 \mu \mathrm{M}$ indomethacin) for 3 days, then switched to WA-differentiation medium (WA-induction medium without dexamethasone) and changed every 2 days, until the preadipocytes differentiated into mature adipocytes. For BA-induction, the procedure was same as WA-induction with the difference of BA-induction medium (WA-induction supplemented with $1 \mathrm{nM}$ triiodothyronine, $1 \mu \mathrm{M}$ rosiglitazone, without indomethacin) and BAdifferentiation medium (BA-induction medium supplemen-

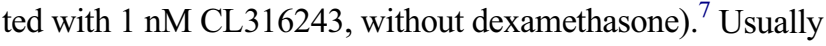
at the 14th day after adipogenic induction, adipocytes became fully differentiated and the typical phenotype appeared. 


\section{Quantitive Real-Time PCR}

Total RNA was isolated from adipocytes using Trizol reagents (Takara, Japan) following the manufacturer's protocol. The quantification of RNA samples was inspected by a Nanodrop 2000 (Thermo Scientific, USA). cDNA synthesis was performed with PrimeScript RT Reagent Kit (Takara Japan) and PCR amplification were carried out with SYBR Green Master Mix (Takara, Japan). Quantitive real-time polymerase chain reaction qRTPCR) was performed using ABM 7500 sequence detection (Biosystems, Foster City, CA, USA), and the gene primers used in this part are shown in Table 1.

\section{Western Blotting Analysis}

Cell protein lysates were obtained from a cocktail with RIPA lysis buffer plus protease-inhibitor ( $\mathrm{Vol} / \mathrm{Vol} 100 / 1)$ in it after 30 minutes on ice, then centrifuged to get the protein sample solution. The protein samples were validated using BCA assay Kit (Thermo Fisher, USA), then denatured at $95^{\circ} \mathrm{C}$ for 10 minutes in loading buffer. $50 \mu \mathrm{g}$ protein samples were loaded and run on a $10 \%$ SDS-polyacrylamide gel, then transfer membrane and immunoblotted with primary antibodies against UCP-1 (1:1000, CST, USA) and beta actin (1:1000, CST, USA), after incubated with HRP (Beijing Zhongshan Gold Bridge Biotechnology, Beijing, China) for $1 \mathrm{~h}$ at room temperature, the protein bands were detected using FluorChemE system (Cell Biosciences, Santa Clara) and the protein bands density were measured using Image J software (v1.8.0).

\section{Lipolysis Analysis}

At the last day of differentiation, $1 \mu \mathrm{M}$ norepinephrine was added into the differentiation medium for 6 hours for both BA- and WA-induced adipocytes and the supernatant of medium was collected. The glycerol concentration in the supernatant was determined using
Adipolysis ELISA Kit (Bioassay, USA), following the manufacturer's recommendation and normalized by the protein content in the well.

\section{Oxygen Consumption Assays}

ADSCs derived from adipose tissue were directly seeded in the plate and induced to differentiation with the WA- or BAinduction procedure using the methods described previously. At day 14 of differentiation, adipocyte oxygen consumption was measured at $37^{\circ} \mathrm{C}$ using the Seahorse XF24-3 extracellular flux analyzer (Agilent, USA). Adipocytes were incubated in XF-assay medium containing $25 \mathrm{mM}$ of glucose, 1 $\mathrm{mM}$ of sodium pyruvate and $2 \mathrm{mM}$ of GlutMAX. Basal oxygen consumption rate (OCR) measurements were taken before sequential addition of reagents, $5 \mu \mathrm{M}$ FCCP was added to render the maximal OCR. OCR values were normalized by the total cell protein concentration measured by BCA Protein Assay Kit (Beyotime, China).

\section{Statistics}

The quantitative physical characteristics and clinical measurements of the NW and OB groups were analyzed by Student's $t$-tests; the gender difference between the NW and OB groups were analyzed by $\mathrm{X}^{2}$ test. In the WA and BA treatment group, the difference of UCP-1 and other thermogenic biomarkers between the NW and OB groups were analyzed by paired two-tailed Student's $t$-tests. Comparisons between WA and BA induction treatment data within the same treatment group were analyzed by analyses of variances (ANOVA). The calculations were performed by SPSS for Windows (version 22.0 software; IBM Corporation, Chicago, IL, USA). Results are expressed as mean $\pm \mathrm{SD}$, and differences between groups were considered statistically significant at $P<0.05$.

Table I The Primer Sequences of Thermogenic and Adipogenic Genes

\begin{tabular}{|l|l|l|}
\hline Genes & Forward & Reverse \\
\hline UCP-I & 5'-GTGCCCAACTGTGCAATGAA-3' & 5'-CCAGGATCCAAGTCGCAAGA-3' \\
PRDMI6 & 5'-GCTGGCAGCTGGCTCAAGTA-3' & 5'-CCTCACCTGGCTCAATGTCCTTA-3' \\
PGC-I $\alpha$ & 5'-TGGTGGACACGAGGAAAGG-3' & 5'-AAATCTGCCCCTGCCAATC-3' \\
FGF2I & 5'-ACAGATGATGCCCAGCAGACAG-3' & 5'-GGATGTCTTGACTCCCAAGATTTGA-3' \\
PPAR $\gamma$ & 5'-CACATTACGAAGACATTCCATTCAC-3' & 5'-GGAGATGCAGGCTCCACTTTG-3' \\
Resistin & 5'-TCTAGCAAGACCCTGTGCTCCA-3' & 5'-ACTCCAGGCCAATGCTGCTTA-3' \\
CIDEA & 5'-TTTCAGACCTTGGGAGACAACAC-3' & 5'-GACTCTCGCTATTCCCGACCT-3' \\
I8S & 5'-GCAATTATTCCCCATGAACG-3' & 5'-GGCCTCACTAAACCATCCAA-3' \\
\hline
\end{tabular}




\section{Results}

\section{Characteristics of Participants}

The anthropological and clinical features of the participants are shown in Table 2. Adipose tissue samples were collected from 24 individuals, and the participants were divided into NW and $\mathrm{OB}$ groups according to their BMI as described in the methods; the two groups were matched for age and sex $(P>0.05)$. The BMI, waist circumference, abdominal visceral and subcutaneous fat area, low-density lipoprotein cholesterol and triglyceride was higher $(P<0.05)$, and high-density lipoprotein cholesterol levels was lower $(P<0.05)$ in the OB group compared to those in the NW group. There was no significant difference in resting metabolic rate, total cholesterol, or blood pressure between the OB and NW groups.

\section{UCP-I Protein Expression in Subcutaneous Adipose Tissue of NW and OB Group}

The result of immunostaining for beige adipocyte marker protein UCP-1 in adipose tissue (Figure 1) showed UCP-1 protein expression in subcutaneous adipose tissue of the NW group was higher compared to that in the OB group. This result is in accordance with previous in-vivo studies which showed that hBAT activity detected by 18 F-PET-CT decreased in obese individuals.

\section{Morphology of WA- and BA-Induced Adipocytes in NW and OB Groups}

We used Oil-red $\mathrm{O}$ staining to examine the morphology of WA- and BA-induced adipocytes in the NW and OB groups and the results showed that the adipocytes in both groups were fully differentiated into mature adipocytes with lipid droplets in the cell staining red. The WAinduced adipocytes in both NW and OB groups contained larger lipid droplets than BA-induced adipocytes, and BAinduced adipocytes contained smaller multinodular lipid droplets in cells. In addition, in BA-induced differentiated adipocytes, there were more cells characterized by multinodular lipid droplets beige-like adipocytes in the NW group than in the OB group (Figure 2).

\section{Thermogenic and Adipogenic Genes Expression in WA- and BA-Induced Adipocytes of NW and OB Group}

Next, we detected the expression of thermogenic and adipogenic genes in WA- and BA-induced adipocytes of NW and OB groups to explore the possible molecular

Table 2 Anthropometric and Biochemical Characteristics of the Participants

\begin{tabular}{|c|c|c|}
\hline & NW Group & OB Group \\
\hline$N$ & 12 & 12 \\
\hline $\operatorname{Sex}(M / F)$ & $4 / 8$ & $5 / 7$ \\
\hline Age (years) & $42.5 \pm 6.4$ & $46.25 \pm 8.47$ \\
\hline BMI $\left(\mathrm{kg} / \mathrm{m}^{2}\right)$ & $21.77 \pm 1.53^{\#}$ & $29.11 \pm 1.22$ \\
\hline Waist circumference $(\mathrm{cm})$ & $76.5 \pm 4.25^{\#}$ & $100.3 \pm 6.99$ \\
\hline Resting metabolic rate $(\mathrm{kcal} / \mathrm{d})$ & $1325.75 \pm 73.75$ & $1225.75 \pm 128.98$ \\
\hline Abdominal subcutaneous fat area $\left(\mathrm{cm}^{2}\right)$ & $175.18 \pm 15.72^{\#}$ & $226.3 \pm 22.24$ \\
\hline Abdominal visceral fat area $\left(\mathrm{cm}^{2}\right)$ & $56.59 \pm 6.5^{\#}$ & $80.31 \pm 8.89$ \\
\hline Diastolic blood pressure $(\mathrm{mmHg})$ & $77 \pm 15.34$ & $82.65 \pm 12.42$ \\
\hline Systolic blood pressure $(\mathrm{mmHg})$ & $121.25 \pm 15.68$ & $135.54 \pm 18.04$ \\
\hline Low-density lipoprotein cholesterin (mmol/L) & $2.59 \pm 0.63 *$ & $3.42 \pm 0.6$ \\
\hline High-density lipoprotein cholesterin $(\mathrm{mmol} / \mathrm{L})$ & $1.37 \pm 0.28^{\#}$ & $1.01 \pm 0.18$ \\
\hline Triglyceride (mmol/L) & $1.1 \pm 0.48^{*}$ & $1.7 \pm 0.82$ \\
\hline
\end{tabular}

Notes: Data are shown as mean \pm SD for continuous variables. $*_{p}<0.05$ and ${ }^{\#} p<0.01$ vs OB group.

Abbreviations: NW, normal weight; OB, obese. 
A

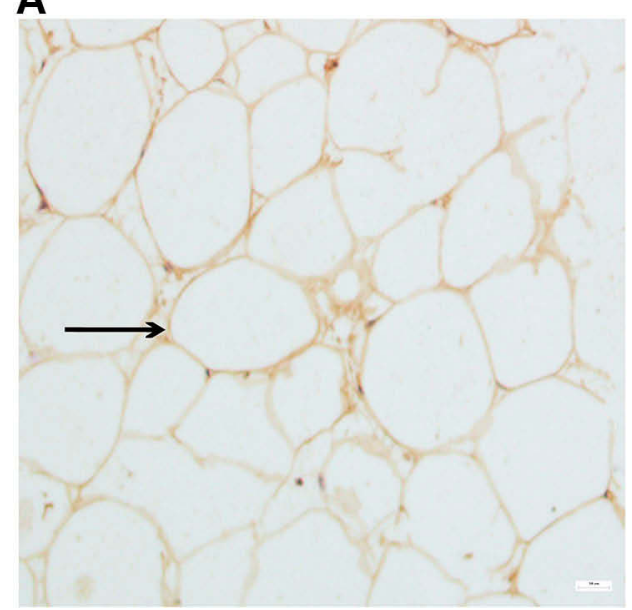

B

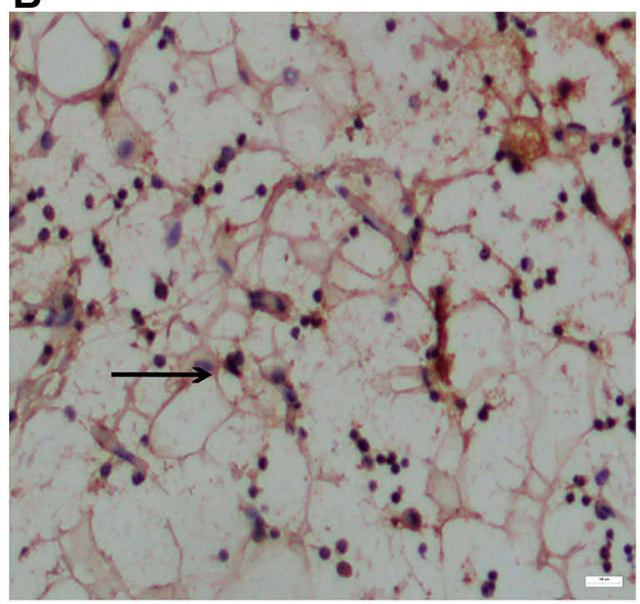

Figure I Immunostaining images of UCP-I expression in adipose tissue derived from obese (OB) (A) and normal weight (NW) group (B). Black arrows suggest the adipocyte size and morphology. Scale bar: $50 \mu \mathrm{m}$.

mechanism involved in impaired hBAT activity in obesity. As shown in Figure 3, the expressions of beige adipocytespecific genes, including UCP-1 (49.24 \pm 8.64 vs $1.08 \pm$ $0.56, P<0.01)$, CIDEA $(3.04 \pm 1.88$ vs $0.95 \pm 0.68, P=$ $0.013)$, PGC-1 $\alpha(14.67 \pm 6.42$ vs $1.05 \pm 0.3, P<0.01)$, PPAR $\gamma(1.99 \pm 0.78$ vs $1.06 \pm 0.35, P<0.01)$, PRDM16 (2.04 \pm 0.96 vs $1.02 \pm 0.7, P=0.037)$ and FGF-21 $(2.5 \pm$ 0.25 vs $1.03 \pm 0.33, P<0.01)$ were significantly higher, and white adipogenic gene resistin was significantly lower $(P<0.01)$ in BA-induced compared to WA-induction adipocytes derived from NW group. But in the OB group, only the expression of CIDEA $(0.54 \pm 0.41$ vs $0.013 \pm$ $0.01, P<0.01)$, PGC- $1 \alpha(0.84 \pm 0.53$ vs $0.2 \pm 0.09, P<$ $0.01)$ were higher in BA-induced adipocytes compared to WA-induced ones, the fold changes in PGC-1 $\alpha$ were lower than in the NW group. There were no significant differences in the expression of UCP-1, PPAR $\gamma$, PRDM16 and FGF-21 between BA-induced and WAinduced adipocytes in OB group. Resistin was also lower in BA-induced compared to WA-induced adipocytes $(P<$ $0.01)$ in the OB group. In either BA- or WA-induced adipocytes, the expression of all the brown/beige adipocyte-specific genes were significantly higher in the NW group than in the OB group. However, the expression of resistin was not different between NW and OB group.

\section{UCP-I Protein Expression in Differentiated Adipocytes from NW and OB Groups}

We also examined the expression of beige/brown adipocytes marker protein UCP-1 in BA-and WA-induced adipocytes by Western blot. The results showed that UCP-1 protein expression levels in adipocytes of the NW group were higher compared that of the OB group, in either WA-induced $(1.45 \pm 0.27$ vs $0.94 \pm 0.34, P<0.01)$ or BA-induced adipocytes $(2.62 \pm 0.51$ vs $1.05 \pm 0.2, P<$ 0.01) (Figure 4A and B). BA-induced adipocytes expressed higher levels of UCP-1 protein than WAinduced adipocytes in the NW group, but there was no significant difference in UCP-1 protein expression between BA- and WA-induced adipocytes in the OB group (Figure 4C), which was in accordance with the results of UCP-1 gene expression level. We characterized the fold changes of UCP-1 protein expression levels (Figure 4D), and found that UCP-1 expression was 1.8-fold in BA-induced compared to WA-induced in the NW group, and 1.1-fold in the OB group. In WA-induced adipocytes, UCP-1 protein expression was 1.5-fold higher in the NW group compared to the OB group, and the changes increased to 2.5-fold in beige-induced adipocytes (Figure 4E).

\section{Lipolysis Analysis of Differentiated Adipocytes of NW and OB Group}

Lipolysis, measured as glycerol release rate, was not significantly different between the NW and OB groups at basal conditions (data not shown). However, a significant increase in lipolysis rate was found in both NW and $\mathrm{OB}$ groups $(P<0.05)$ after norepinephrine $(1 \mu \mathrm{M})$ treatment for $6 \mathrm{~h}$; the norepinephrine-induced lipolysis was not significantly different between WA- and BA-induced adipocytes in the OB group, but the glycerol concentration of 

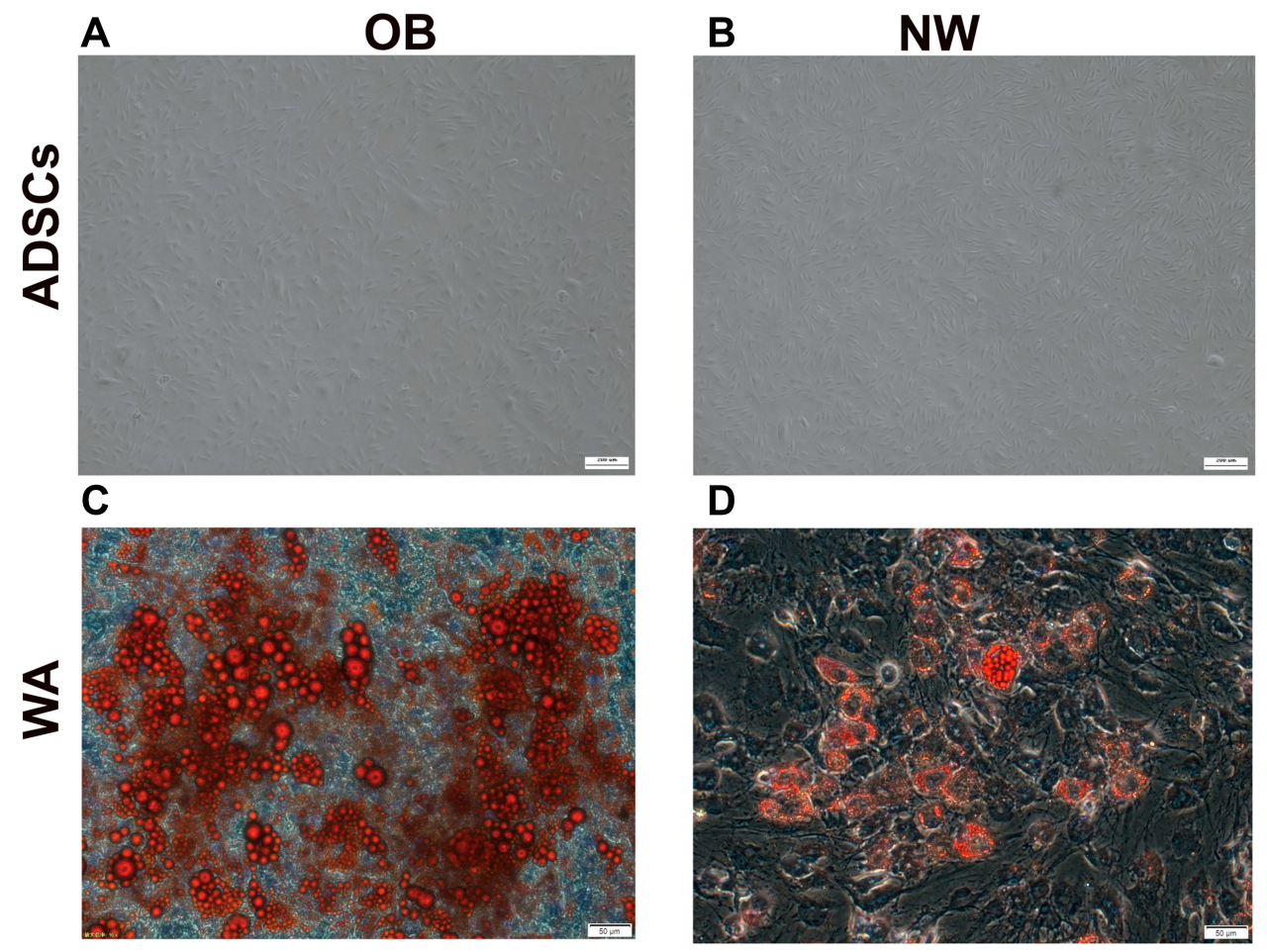

$\mathbf{E}$

$\mathbf{F}$
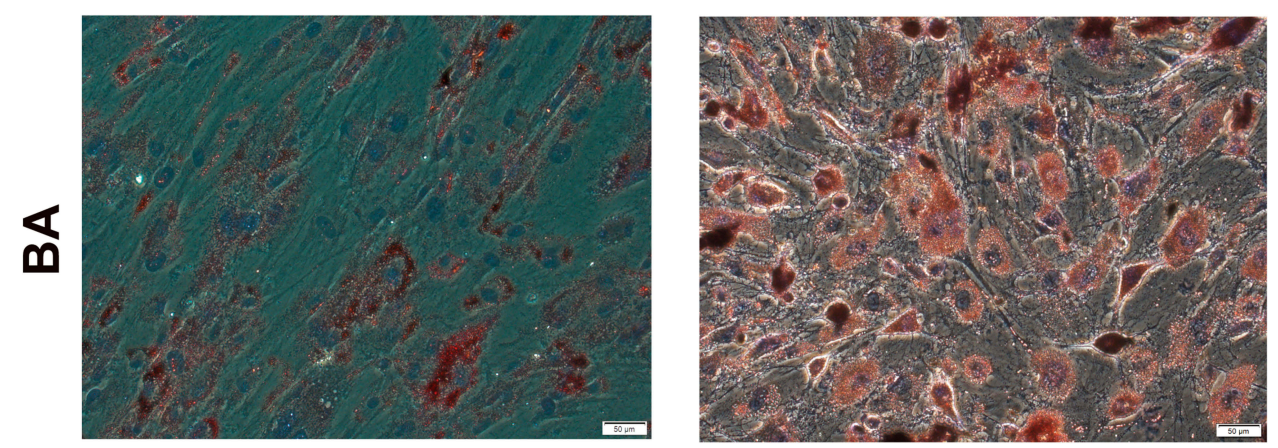

Figure 2 Human adipose-derived stem cells (ADSCs) were induced to differentiate into mature adipocytes. (A, C and $\mathbf{E})$ show the cell images of the obese (OB) group. (B, $\mathbf{D}$ and $\mathbf{F})$ show the cell photo of the normal-weight (NW) group. (A and $\mathbf{B})$ : ADSCs; (C and $\mathbf{D})$ : adipocytes with white adipocyte (WA)-induction treatment (I4th day). $(\mathbf{E}$ and $\mathbf{F})$ : adipocytes with beige adipocytes (BA)-induction treatment (I4th day). Oil red $\mathrm{O}$ staining image showed lipid droplet in differentiated adipocytes. Scale bar: $50 \mu \mathrm{m}$ for differentiated adipocytes, $200 \mu \mathrm{m}$ for ADSCs.

BA-induced adipocytes was higher than WA-induced adipocytes in the NW group $(3.65 \pm 0.78$ vs $2.32 \pm 0.42 \mu \mathrm{g} /$ $\mathrm{mL} / \mu \mathrm{g}$ protein, $P<0.01$; Figure 5 ). And the lipolysis ability of the NW group was higher than the OB group, in either WA- $(2.32 \pm 0.42$ vs $0.67 \pm 0.23 \mu \mathrm{g} / \mathrm{mL} / \mu \mathrm{g}$ protein, $P<0.01)$ or BA-induced adipocytes (3.65 \pm 0.78 vs $0.97 \pm 0.18 \mu \mathrm{g} / \mathrm{mL} / \mu \mathrm{g}$ protein, $P<0.01$; Figure 5).

\section{Functional Analysis of Mitochondrial Respiration in Differentiated Adipocytes}

To assay the mitochondrial respiration function of fat cells with different induction procedures from individuals of different adiposity, we investigated the mitochondrial oxygen consumption rate (OCR) of differentiated adipocytes of the four groups (Figure 6). At a functional level, the basal and maximum respiratory OCRs of BA-induced adipocytes were higher than those of WA-induced adipocytes in both NW (132.3 \pm 4.69 vs $108.82 \pm 4.09 \mathrm{pmol} / \mathrm{min} / \mu \mathrm{g}$ protein, $158.46 \pm 3.97 \mathrm{vs}$ $100.66 \pm 3.59 \mathrm{pmol} / \mathrm{min} / \mu \mathrm{g}$ protein, respectively, $P<$ $0.01)$ and $\mathrm{OB}$ groups $(78.62 \pm 10.3$ vs $57.55 \pm 7.64$ $\mathrm{pmol} / \mathrm{min} / \mu \mathrm{g}$ protein, $76.43 \pm 3.98$ vs $61.96 \pm 5.13$ $\mathrm{pmol} / \mathrm{min} / \mu \mathrm{g}$ protein, respectively, $P<0.01$ ). In the NW group, the basal OCR and maximum OCR went up 1.2- and1.6-fold, respectively, in BA-induced fat 
A

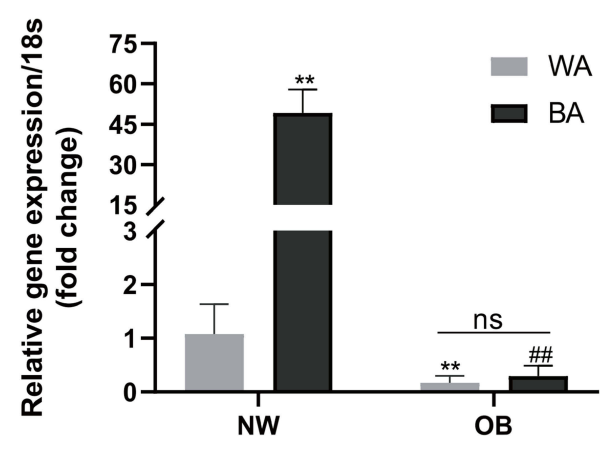

C

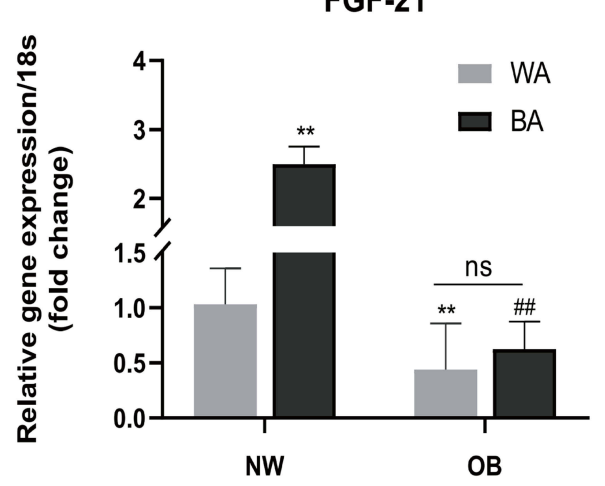

E

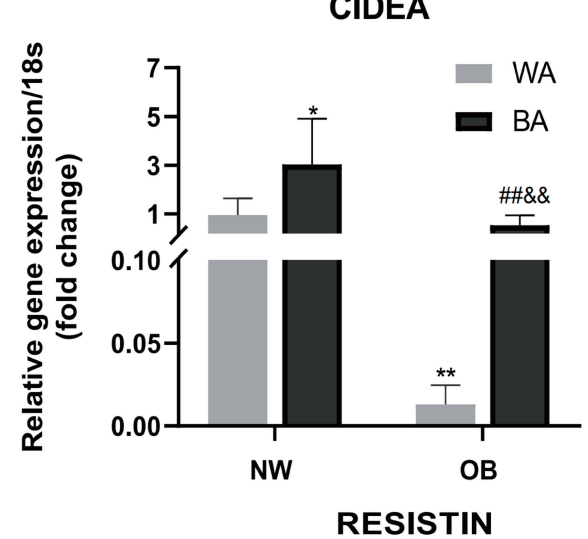

G

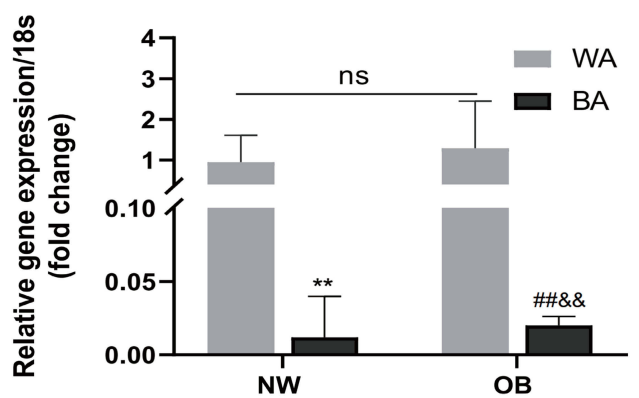

B

PRDM16

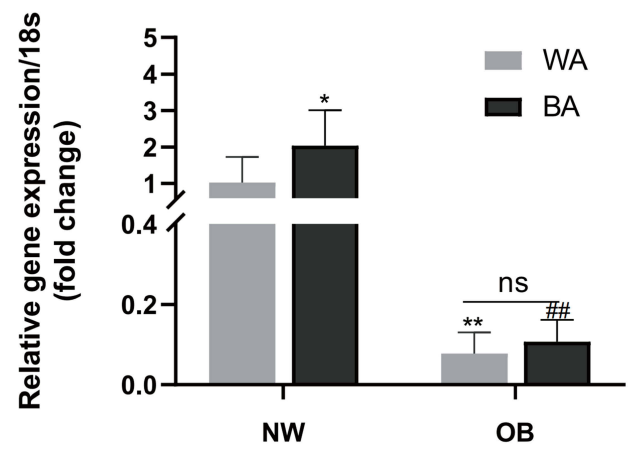

D

PGC-1a

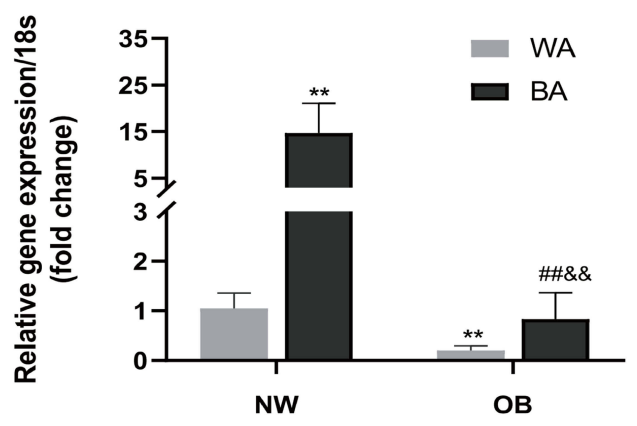

F

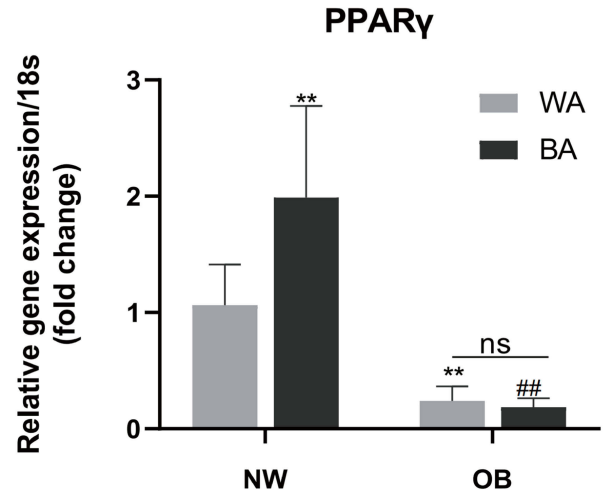

Figure 3 Thermogenic and adipogenic genes expressions in differentiated adipocytes. Comparison of UCP-I (A), PRDMI6 (B), FGF2I (C), PGC-I $\alpha$ (D), CIDEA (E), PPAR $\gamma$ (F) and resistin (G) mRNA expressions of WA- and BA-induced adipocytes in normal-weight (NW) and obese (OB) groups. Values are mean \pm SD; $n=12$ for NW-WA, NW-BA, OB-WA and OB-BA groups; data used for analysis were mean values of three replicate experiments for each sample; $*_{p}<0.05$ vs NW-WA group, **p< 0.01 vs NW-WA group, ${ }^{\ldots} p<0.01$ vs NW-BA group, ${ }^{\text {\&\&}} p<0.01$ vs OB-WA group.

Abbreviation: ns, not significant. 
A

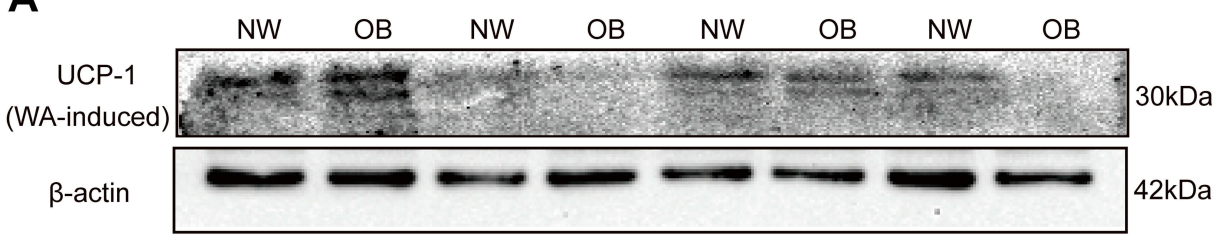

B

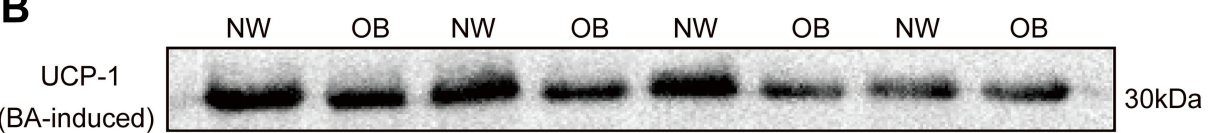
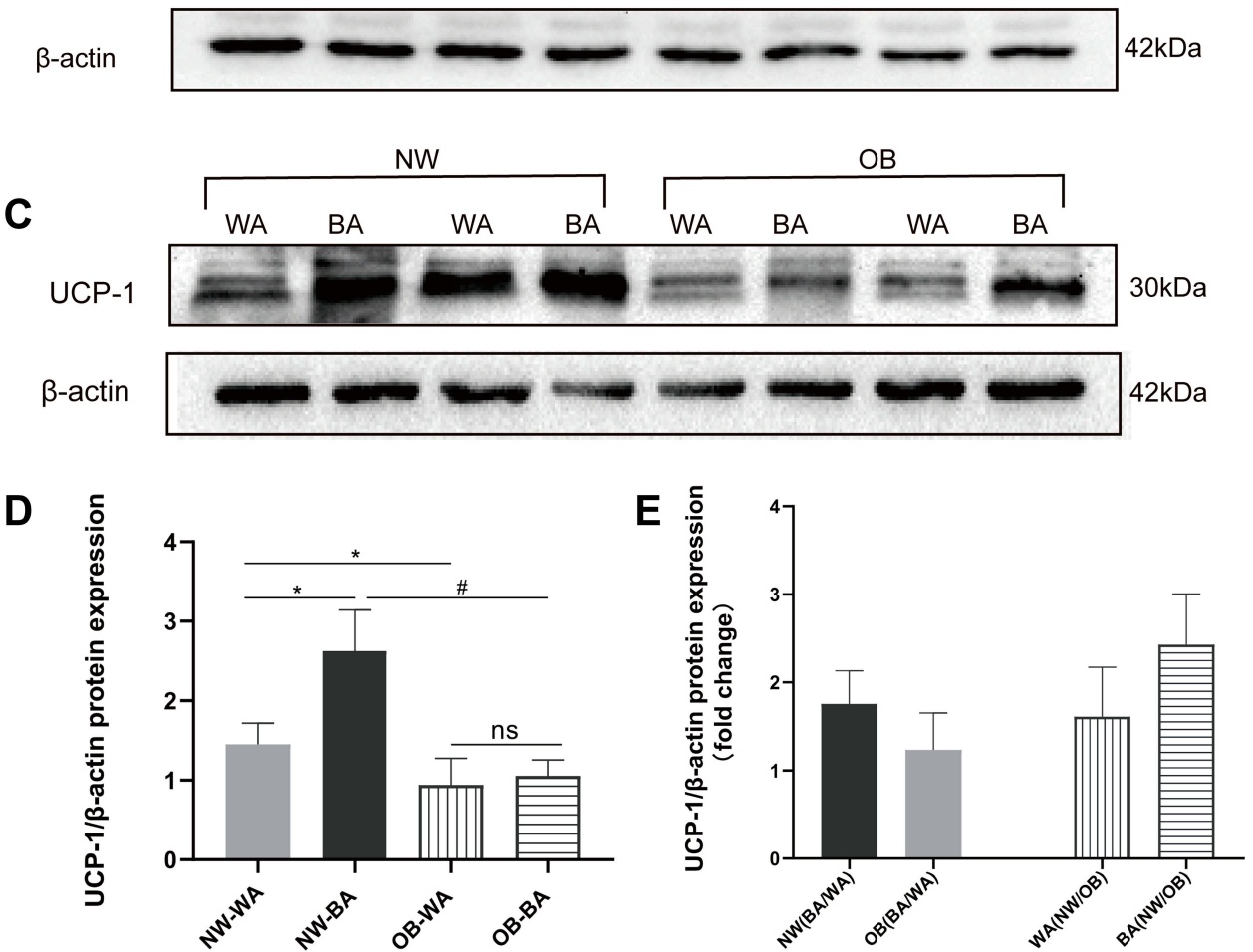

Figure 4 UCP-I protein expression in differentiated adipocytes. Western-blot images of UCP-I protein band density of normal-weight group (NW) and obese (OB) group in WA (white adipocyte)-induced (A) and BA (beige adipocyte)-induced (B) adipocytes, and UCPI protein band density of WA-and BA-induced adipocytes from the same adipose tissue sample in NW and OB group (C). (D) The analysis of UCP-I protein band values in NW-WA, NW-BA, OB-WA and OB-BA groups. (E) Fold changes of UCPI protein expression in NW and OB group with WA- and BA-induction treatment. Values are mean \pm SD; $n=12$ for NW-WA, NW-BA, OB-WA and OB-BA groups; ${ }^{*} p<$ 0.01 vs NW-WA group, ${ }^{\#} p<0.01$ vs NW-BA group.

Abbreviation: ns, not significant.

cells compared to WA-induced adipocytes, and 1.3- and 1.2-fold, respectively, in the OB group. However, adipocytes derived from the $\mathrm{OB}$ group always had lower basal and maximum OCR values compared to those from the NW group, in either BA- or WA-induced adipocytes. These data indicated BA-induction procedure during adipocytes differentiation could increase mitochondrial respiratory function in both $\mathrm{OB}$ and NW groups, which was a characteristic of beige adipocytes, and adipocytes from the NW group always showed a higher respiratory capacity compared to those from the OB group.

\section{Discussion}

The activation of BAT has been a promising therapeutic target for treatment of obesity and related metabolic disease recently. However, a few studies have indicated that obesity is inversely associated with impaired BAT activity in rodents and humans, ${ }^{8-10}$ and the underlying molecular and cellular mechanism remains unclear. The present study also verified that the expression of beige/brown adipocytes marker protein UCP-1 was decreased in adipose tissue of obese persons, which was in accordance with the previous human in-vivo studies. We used human adipocyte culture and BA- and WAinduction treatment to explore the underlying mechanism in 


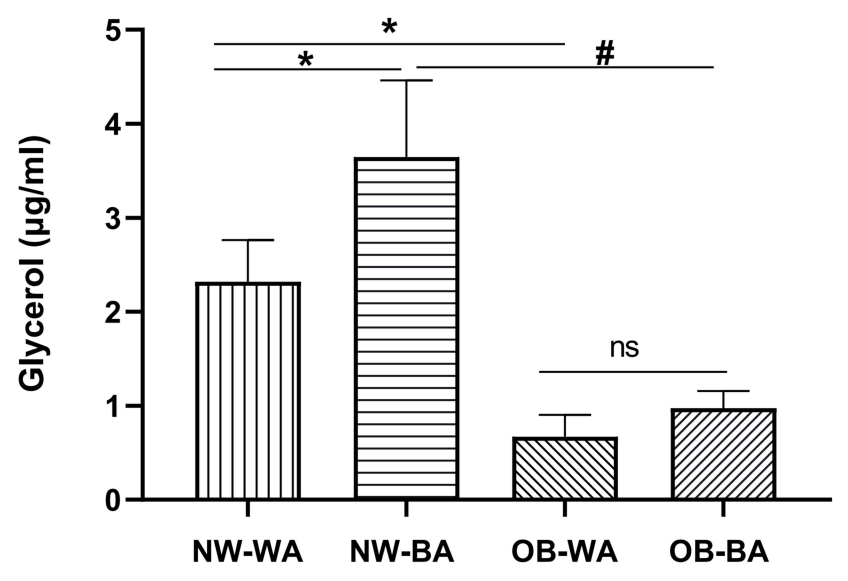

Figure 5 Lipolysis analysis of differentiated adipocytes. Glycerol concentration was measured in the media to determine rates of lipolysis in WA (white adipocyte)- and BA (beige adipocyte)-induced adipocytes derived from NW (normal-weight group) and $O B$ (obese group) after treatment with I $\mu M$ norepinephrine for $6 \mathrm{~h}$. Values are mean \pm SD; $n=12$ for NW-WA, NW-BA, OB-WA and OB-BA groups; data used for analysis are mean values of three replicate experiments for each sample; $* p<$ 0.01 vs NW-WA group, ${ }^{\#} p<0.01$ vs NW-BA group.

Abbreviation: ns, not significant.

the present study, and found that subcutaneous fat cells derived from obese patients had compromised beige adipocytes differentiation potential in Chinese Han race population.

Several human studies used 18F-PET-CT to explore hBAT activity in different race populations including Asians and Caucasians, ${ }^{11-14}$ most of them paved the way for the reduction of BAT activity to be associated with increased adiposity and risk for type 2 diabetes. ${ }^{15}$ It has also been proved that hBAT could be activated by $\beta$ - adrenergic agonist in lean subjects, especially in young and lean females, ${ }^{16}$ but not in obese subjects. The present study used primary human adipocyte culture and beige/white adipogenic induction to differentiate ADSCs into mature adipocytes in vitro, which could mimic the morphological and transcriptional process during adipogenesis and maintained physiological features of human mature adipocytes in vivo, and could provide a valuable model to observe the beige adipocytes potential in people with different adiposity intuitively in vitro. In addition, the present study performed both beige- and white-adipocyte induction treatment to preadipocytes derived from the same adipose tissue sample, which can provide comprehensive cellular information concerning beige/white adipocyte differentiation, and the data in this field were limited so far.

Our data showed that the expression of beige adipocyte marker gene and protein UCP-1 was decreased in adipocytes derived from obese individuals, which was in accordance with the results in human adipose tissue. To detect the underlying mechanism, the present study investigated the beige-specific thermogenic genes expression in BA- and WA-induced differentiated adipocytes derived from persons with different adiposity, and the results showed that the expression of PRDM16, PPAR $\gamma$, PGC-1 $\alpha$, FGF21 and CIDEA were higher in normal-weight individuals compared with obese persons, and BA-induction treatment could significantly increase the expression of beige adipocytespecific genes in adipocytes derived from normal-weight
A

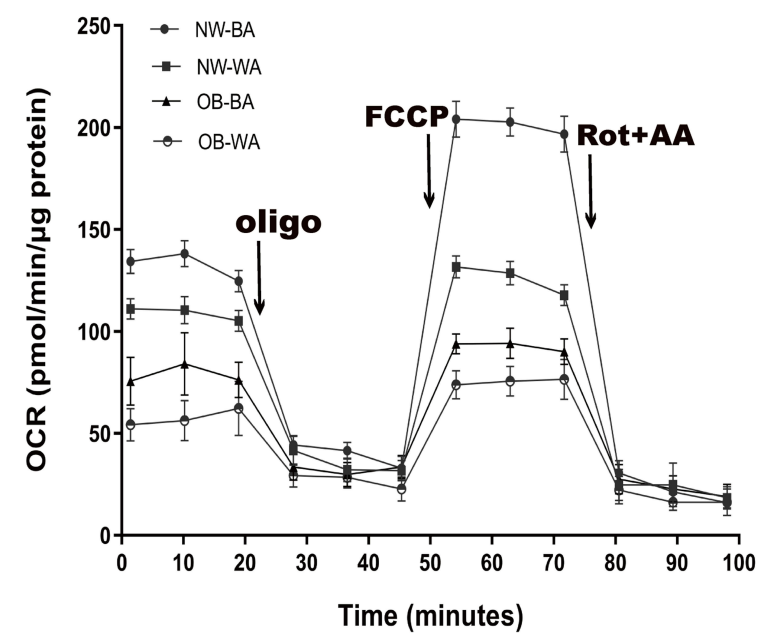

B

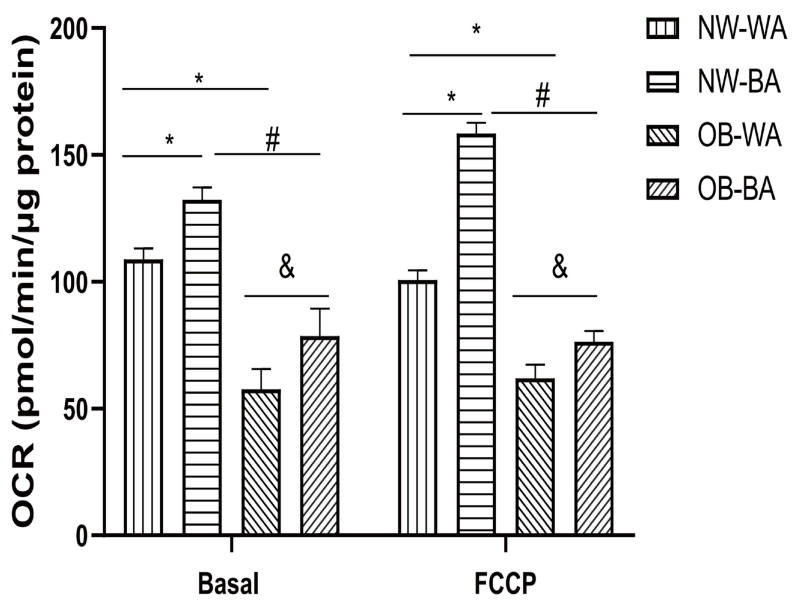

Figure 6 Mitochondria oxygen consumption rate in differentiated adipocytes. The differentiated adipocytes derived from the normal-weight (NW) and obese (OB) groups treated with white adipocyte (WA)- and beige adipocytes (BA)-induction were used to detect oxygen consumption rate (OCR). Basal OCR were measured before sequential addition of reagents, FCCP was added to render maximum OCR $(\mathbf{A})$. Basal and maximum OCR were normalized by the total-cell protein concentration (B). Values are mean $\pm S D ; n=12$ for the NW-WA, NW-BA, OB-WA and OB-BA groups; data used for analysis are mean values of four replicate wells for each sample. * $p<0.0 \mathrm{I}$ vs NW-WA group, ${ }^{\#} p<0.01$ vs NW-BA group, ${ }^{\&} p<0.01$ vs OB-WA group. 
persons, but not in obese ones. These genes are involved in the process of mitochondria biogenesis and drive the ADSCs to differentiate into beige adipocytes, so their low expression during adipocyte differentiation may result in reduced beige adipocyte differentiation potential and compromised hBAT activity in obese persons.

We also measured the adipocyte oxygen consumption rate to assay the mitochondria function of differentiated adipocytes from individuals with different adiposity and the consequence of BA-and WA-induction treatment, and the results showed impaired mitochondrial respiratory function in adipocytes from obese persons. Compared to WA-induction treatment, BA-induction significantly increased the basal and maximum mitochondria oxygen consumption rate in adipocytes of both normal-weight individuals and obese persons, which was not in accordance with the results of the expression UCP-1, where the expression did not change with BA- or WA-induction in obese persons; this phenomenon may due to the UCP-1-independent thermogenic pathway. ${ }^{17,18}$

Lipolysis analysis of differentiated adipocytes also revealed that the fat cells from the obese group had a lower level of lipolysis compared with normal-weight individuals. BA-induction treatment significantly increased the lipolysis ability in adipocytes of normalweight individuals, although there was also an increasement in glycerol release rate in BA-induced adipocytes of obese ones, but the level of increase was lower than that of normal-weight persons. Some researchers have recently found that lipolysis in WAT triggers insulin release, which is essential for the replenishment of BAT energy stores and for efficient adaptive thermogenesis. ${ }^{19}$ So the compromised beige adipocyte potential may be due part to the lower lipolysis ability in obesity.

Because BA-induction treatment can significantly increase the beige adipocytes biomarker gene and protein, thermogenic and lipolysis function in adipocytes was derived from normal-weight persons, not obese ones. So next we analyze the difference in BA- and WA-induction treatments to try to figure out the possible underlying molecular mechanism. As described in the methods, CL316243, triiodothyronine (T3) and rosiglitazone were included in the BA-induction procedure but not in WAinduction, which was the main difference.

In the prevailing model, cold temperatures trigger sympathetic discharge, which results in the release of noradrenaline in brown and white adipose tissue that activates $\beta 3$-adrenergic receptors ( $\beta 3-\mathrm{AR}),{ }^{20}$ noradrenergic signaling induces the expression of thermogenic genes, which is the domain way to increase hBAT activity. CL316243 is a selective $\beta 3$ agonist that could mimic cold and adrenergic simulation. ${ }^{21} \beta 3-\mathrm{AR}$ agonists can stimulate human BAT activity in normal weight adults, but this ability was impaired in people with obesity tested via $18 \mathrm{~F}-\mathrm{FDG}$ PET/CT detection. ${ }^{22}$ The present study proves in vitro that subcutaneous adipocytes can be BAinduced to attain beige adipocyte characteristics, but this capacity was also reduced in adipocytes from obese individuals. A study we published recently showed that adipocytes $\beta 3$-adrenergic receptor ( $\beta 3$-AR) expression was downregulated in obese/overweight individuals, ${ }^{9}$ which may contribute partly to the compromised beige inducible capacity in adipose tissue of obese persons.

Thyroid hormones contribute to BAT thermogenesis and affect energy balance in rodents and humans. ${ }^{23} \mathrm{~T} 3$ promotes the expression of PGC- $1 \alpha$, the master transcriptional coactivator of mitochondrial biogenesis in fat depots to enhance BAT thermogenesis. ${ }^{24}$ The results of an animal study suggested that thyroid hormone also additionally mediates UCP-1-independent thermogenesis. ${ }^{25}$ BAinduction treatment did not increase the expression of UCP-1 in adipocytes derived from the obese group, but mitochondrial respiratory function, PGC- $1 \alpha$ gene expression did increase significantly, which may due to the effect of T3, although the content of increasement was lower compared to normal-weight ones. Further studies should be carried out to elucidate the molecular signaling pathway through which $\mathrm{T} 3$ plays a role in adipocyte thermogenesis.

Rosiglitazone is a potent PPAR $\gamma$ agonist that causes mitochondrial biogenesis and increased expression of several mitochondrial proteins. ${ }^{26}$ The adipocyte expression of PPAR $\gamma$ in obesity was lower than normal-weight ones in the present study, which may contribute partly to the decreased beige inducible potential when facing BAinduction treatment in obesity.

The present study indicated that adipocytes from subcutaneous white-adipose tissue expressed a lower level of beige adipocytes biomarker genes and protein in obese Chinese, followed by reduced mitochondrial respiratory and lipolysis function in adipocytes. ADSCs from the obese group showed a lower beige inducible ability compared to that of normalweight individuals. Sympathetic stimuli could enhance the browning plasticity in ADSCs derived from normal-weight individuals during adipocyte differentiation, but not in obesity in Chinese, which may be due in part to the downregulation of $\beta 3$-AR in adipocytes. Regardless of whether defective BAT is a cause or a consequence of obesity, BAT-activation strategies remain a potential therapeutic target in individuals with 
established obesity. Therefore, the discovery of therapeutic agents to activate BAT through specific pathways could provide a promising approach for treating obesity in the future.

\section{Acknowledgments}

We are thankful to Li Fang for technical assistance with data analysis and to Prof. Qiu for kindly providing adipose tissue.

\section{Funding}

This study was funded by the Natural Science Foundation of China $(81,300,686)$ and the Key Research and Development Program of Shandong Province (2018GSF118184). The sponsors had no influence on the design, conduct, interpretation or publication of this work.

\section{Disclosure}

The authors report no conflicts of interest in this work.

\section{References}

1. Quan L-H, Zhang C, Dong M. Myristoleic acid produced by enterococci reduces obesity through brown adipose tissue activation. Gut. 2020;69(7):1239-1247. doi:10.1136/gutjnl-2019-319114

2. Ruiz JR, Martinez-Tellez B, Sanchez-Delgado G, Osuna-Prieto FJ, Rensen PCN, Boon MR. Role of human brown fat in obesity, metabolism and cardiovascular disease: strategies to turn up the heat. Prog Cardiovasc Dis. 2018;61(2):232-245. doi:10.1016/j.pcad.2018.07.002

3. Chang S-H, Song N-J, Choi JH, Yun UJ, Park KW. Mechanisms underlying UCP1 dependent and independent adipocyte thermogenesis. Obes Rev. 2019;20(2):241-251. doi:10.1111/obr.12796

4. van Rooijen BD, van der Lans AAJJ, Brans B. Imaging cold-activated brown adipose tissue using dynamic $\mathrm{T} 2 *$-weighted magnetic resonance imaging and 2-deoxy-2-[18F]fluoro-D-glucose positron emission tomography. Invest Radiol. 2013;48(10):708-714. doi:10.1097/ RLI.0b013e31829363b8

5. Martinez-Tellez B, Xu H, Sanchez-Delgado G. Association of wrist and ambient temperature with cold-induced brown adipose tissue and skeletal muscle [18F]FDG uptake in young adults. Am J Physiol Regul Integr Comp Physiol. 2018;315(6):R1281-R1288. doi:10.1152/ ajpregu.00238.2018

6. Strieder-Barboza C, Thompson E, Thelen K, Contreras GA. Technical note: bovine adipocyte and preadipocyte co-culture as an efficient adipogenic model. J Dairy Sci. 2019;102(4):3622-3629. doi:10.3168/ jds.2018-15626

7. Lee P, Werner CD, Kebebew E, Celi FS. Functional thermogenic beige adipogenesis is inducible in human neck fat. Int $J$ Obes. 2014;38 (2):170-176. doi:10.1038/ijo.2013.82

8. Hui X, Gu P, Zhang J. Adiponectin enhances cold-induced browning of subcutaneous adipose tissue via promoting M2 macrophage proliferation. Cell Metab. 2015;22(2):279-290. doi:10.1016/j. cmet.2015.06.004

9. Cao W-Y, Liu Z, Guo F, Yu J, Li H, Yin X. Adipocyte ADRB3 down-regulated in Chinese overweight individuals adipocyte ADRB3 in overweight. Obes Facts. 2018;11(6):524-533. doi:10.1159/ 000495116
10. Garcia RA, Roemmich JN, Claycombe KJ. Evaluation of markers of beige adipocytes in white adipose tissue of the mouse. Nutr Metab. 2016;13(1):24. doi:10.1186/s12986-016-0081-2

11. Crandall JP, Gajwani P, Joo HO, Mawhinney DD, Sterzer F, Wahl RL. Repeatability of brown adipose tissue measurements on FDG PET/CT following a simple cooling procedure for BAT activation. PloS one. 2019;14(4):e0214765. doi:10.1371/journal.pone.0214765

12. Deng J, Neff LM, Rubert NC, et al. MRI characterization of brown adipose tissue under thermal challenges in normal weight, overweight and obese young men. Journal of magnetic resonance imaging : JMRI. 2018;47(4):936-947. doi:10.1002/jmri.25836

13. Sanchez-Delgado G, Martinez-Tellez B, Olza J, et al. Activating brown adipose tissue through exercise (ACTIBATE) in young adults: Rationale, design and methodology. Contemporary clinical trials. 2015;45:416-425. doi:10.1016/j.cct.2015.11.004

14. Bakker LEH, Boon MR, van der Linden RAD. Brown adipose tissue volume in healthy lean south Asian adults compared with white Caucasians: a prospective, case-controlled observational study. Lancet Diabetes Endocrinol. 2014;2(3):210-217. doi:10.1016/ s2213-8587(13)70156-6

15. Censin JC, Peters SAE, Bovijn J. Causal relationships between obesity and the leading causes of death in women and men. PLoS Genet. 2019;15(10):e1008405. doi:10.1371/journal.pgen.1008405

16. van den Beukel JC, Grefhorst A, Hoogduijn MJ. Women have more potential to induce browning of perirenal adipose tissue than men. Obesity. 2015;23(8):1671-1679. doi:10.1002/oby.21166

17. Grimpo K, Völker MN, Heppe EN, Braun S, Heverhagen JT, Heldmaier G. Brown adipose tissue dynamics in wild-type and UCP1-knockout mice: in vivo insights with magnetic resonance. J Lipid Res. 2014;55(3):398-409. doi:10.1194/jlr.M042895

18. Olsen JM, Csikasz RI, Dehvari N. $\beta$-adrenergically induced glucose uptake in brown adipose tissue is independent of UCP1 presence or activity: mediation through the mTOR pathway. Mol Metab. 2017;6 (6):611-619. doi:10.1016/j.molmet.2017.02.006

19. Heine M, Fischer AW, Schlein C. Lipolysis triggers a systemic insulin response essential for efficient energy replenishment of activated brown adipose tissue in mice. Cell Metab. 2018;28(4):644-655.e4. doi:10.1016/j.cmet.2018.06.020

20. Liu P, Huang S, Ling S. Foxp1 controls brown/beige adipocyte differentiation and thermogenesis through regulating $\beta 3$-AR desensitization. Nat Commun. 2019;10(1):5070. doi:10.1038/s41467-01912988-8

21. Cypess A, Weiner L, Roberts-Toler C. Activation of human brown adipose tissue by a $\beta 3$-adrenergic receptor agonist. Cell Metab. 2015;21(1):33-38. doi:10.1016/j.cmet.2014.12.009

22. Carey AL, Formosa MF, Van Every B. Ephedrine activates brown adipose tissue in lean but not obese humans. Diabetologia. 2013;56 (1):147-155. doi:10.1007/s00125-012-2748-1

23. Skarulis MC, Celi FS, Mueller E. Thyroid hormone induced brown adipose tissue and amelioration of diabetes in a patient with extreme insulin resistance. J Clin Endocrinol Metab. 2010;95(1):256-262. doi:10.1210/jc.2009-0543

24. Bae IS, Kim SH.Expression and secretion of an atrial natriuretic peptide in beige-like 3T3-L1 adipocytes. Int J Mol Sci. 2019;20 (24):6128. doi:10.3390/ijms20246128

25. Dittner C, Lindsund E, Cannon B, Nedergaard J. At thermoneutrality, acute thyroxine-induced thermogenesis and pyrexia are independent of UCP1. Mol Metab. 2019;25:20-34. doi:10.1016/j. molmet.2019.05.005

26. Fayyad AM, Khan AA, Abdallah SH, Alomran SS, Bajou K, Khattak MN. Rosiglitazone enhances browning adipocytes in association with MAPK and PI3-K pathways during the differentiation of telomerase-transformed mesenchymal stromal cells into adipocytes. Int J Mol Sci. 2019;20(7). doi:10.3390/ijms20071618 


\section{Publish your work in this journal}

Diabetes, Metabolic Syndrome and Obesity: Targets and Therapy is an international, peer-reviewed open-access journal committed to the rapid publication of the latest laboratory and clinical findings in the fields of diabetes, metabolic syndrome and obesity research. Original research, review, case reports, hypothesis formation, expert opinion and commentaries are all considered for publication. The manuscript management system is completely online and includes a very quick and fair peer-review system, which is all easy to use. Visit http://www.dovepress.com/testimonials.php to read real quotes from published authors.

Submit your manuscript here: https://www.dovepress.com/diabetes-metabolic-syndrome-and-obesity-targets-and-therapy-journal 